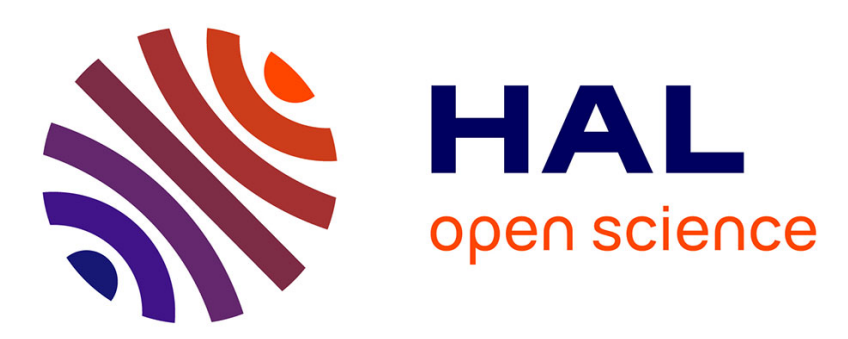

\title{
Camera model identification based on the generalized noise model in natural images
}

\author{
Thanh Hai Thai, Florent Retraint, Rémi Cogranne
}

\section{To cite this version:}

Thanh Hai Thai, Florent Retraint, Rémi Cogranne. Camera model identification based on the generalized noise model in natural images. Digital Signal Processing, 2016, 48, pp.285-297. 10.1016/j.dsp.2015.10.002 . hal-01915650

\section{HAL Id: hal-01915650 \\ https://hal.science/hal-01915650}

Submitted on 15 Mar 2019

HAL is a multi-disciplinary open access archive for the deposit and dissemination of scientific research documents, whether they are published or not. The documents may come from teaching and research institutions in France or abroad, or from public or private research centers.
L'archive ouverte pluridisciplinaire HAL, est destinée au dépôt et à la diffusion de documents scientifiques de niveau recherche, publiés ou non, émanant des établissements d'enseignement et de recherche français ou étrangers, des laboratoires publics ou privés. 


\title{
Camera Model Identification Based on the Generalized Noise Model in Natural Images
}

\author{
Thanh Hai Thai ${ }^{\mathrm{a} *}$, Florent Retraint ${ }^{\mathrm{b}}$ and Rémi Cogranne $\mathrm{e}^{\mathrm{b} * *}$ \\ a Institue of Research and Development, Duy Tan University, Danang, Vietnam \\ ${ }^{b}$ ICD - LM2S - ROSAS - UMR 6281 CNRS, Troyes University of Technology, 12 rue Marie Curie, 10010 Troyes cedex, France
}

\begin{abstract}
The goal of this paper is to design a statistical test for the camera model identification problem. The approach is based on a generalized noise model that is developed by following the image processing pipeline of the digital camera. More specifically, this model is given by starting from the heteroscedastic noise model that describes the linear relation between the expectation and variance of a RAW pixel and taking into account the non-linear effect of gamma correction. The generalized noise model characterizes very accurately a rendered natural image after incamera post-acquisition processes up to the JPEG compression. In the present paper the parameters that are specified in the generalized noise model are used as camera fingerprint to identify camera models from a digital image. The camera model identification problem is cast in the framework of hypothesis testing theory. In an ideal context where all model parameters are perfectly known, the Likelihood Ratio Test is presented and its statistical performances are theoretically established. In practice when the model parameters are unknown, two Generalized Likelihood Ratio Tests are designed to deal with this difficulty such that they can meet a prescribed false alarm probability while ensuring a high detection performance. Numerical results on simulated images and real natural JPEG images highlight the relevance of the proposed approach.
\end{abstract}

Keywords: Digital Forensics, Camera Model Identification, Hypothesis Testing, Natural Image Model, Nuisance Parameters.

\footnotetext{
${ }^{*}$ Corresponding author.

***With the financial support from Champagne-Ardenne region, IDENT project.

Email address: thanh_hai.thai.2014@utt.fr (Thanh Hai Thai ${ }^{\mathrm{a}}$ )
} 


\section{Introduction}

Digital forensics has received a great attention from law enforcement agencies and academic researchers in the past decade. Because of dramatic advancement in computing and network technologies, the accessibility and transmission of digital images have been increased remarkably. Digital images can be easily edited, altered or falsified because of the large availability of image editing software tools. Consequently, the reliability and trustworthiness of digital images have been questioned when used as evidence in legal and security domains. Reliable forensic methods are urgently needed by law enforcement agencies to restore the trust to digital images.

\subsection{State of the Art}

Generally, digital image forensics involves two key problems: image origin identification and image forgery detection (see [1] and the references therein for a detailed introduction). The problem of image origin identification aims at verifying whether a given image was acquired with a specific camera, or at determining the camera model/brand as well as types of imaging mechanism (e.g. scanners, cell-phone cameras, or computer graphics). The image forgery detection aims at detecting any act of manipulation such as splicing, removal or adding in an image. There are two approaches to address these problems. Active approach such as digital signatures [2] and digital watermarking [3] has some limitations because a dedicated information has to be embedded during the creation of an image, which increases the production cost of digital cameras, and the credibility of information embedded in the image remains questionable. Passive approaches has been increasingly studied in the past decade since it does not impose any constraint and does not require any prior information. Forensic analysts have only the suspect image at their disposal and must explore useful information from that image to gather forensic evidence, trace the acquisition device and detect any act of manipulation. Passive approaches are based on internal traces left by the camera in a given image. These internal traces can be provided by investigating the image acquisition pipeline; see [4, 5] for an overview of the structure and processing steps of a typical digital camera. Every step from real-world scene acquisition to image storage can provide clues for forensic analysis.

In image origin identification problem, it is important to distinguish the problem of camera instance identification and the problem of camera model/brand identification. More specifically, fingerprints used for camera instance identification should capture individuality, especially different cameras of the very same model. For camera model/brand identification, it is necessary to exploit fingerprints that are shared between cameras of the same model/brand but discriminative for different any other camera models/brands. The present proposes a novel method that belong to the passive forensic methods for which prior-arts can be divided into two categories.

Methods in the first category rely on the assumption that there are differences in image processing techniques and component technologies between different camera models. Lens aberration [6], Color Filter Array (CFA) patterns with interpolation algorithms $[7,8,9,10]$ and JPEG compression parameters [11] are considered as influential factors for camera model identification. Using these factors, a forensic feature set is provided and used in a machine 
learning algorithm. The main challenge for this approach is that the image processing techniques remain identical or similar, and the components produced by a few manufacturers are shared among camera models. Moreover, as in all applications of machine learning, it is difficult to select an accurate feature set.

Methods in the second category aim at identifying unique characteristics or fingerprints of the acquisition camera device. Sensor Pattern Noise (SPN) is caused by imperfections during the manufacturing process and non-uniformity of photo-electronic conversion due to inhomogeneity of silicon wafers. This is the unique fingerprint which the methods are mainly based on to identify the camera device. The reader is referred to [12] for the first version of this work and $[13,14,15]$ for the improved version. Two main components of the SPN are the Fixed Pattern Noise (FPN) and the Photo-Response Non-Uniformity (PRNU) noise. The FPN used in [16] for camera device identification can be compensated by subtracting a dark frame from the output image. Therefore, the FPN is not a robust fingerprint and no longer used in later works. The PRNU, which is directly exploited in $[13,14,15]$, can be also used for camera model identification as proposed in [17] based on the assumption that that fingerprint obtained from images in the TIFF or JPEG format contains traces of post-acquisition processes (e.g. demosaicing) that carry information about the camera model. The ability to extract this noise reliably from a given image is the main challenge in this category due to interference of non-unique operations (e.g. demosaicing and JPEG compression).

\subsection{Main Contributions of the Paper}

The present paper addresses the problem of camera model identification using a passive approach. In the literature, a vast majority of prior work are based on machine learning methods to design a classifier that distinguish camera models. The main drawback is that this framework requires an expensive training stage that comprises many images with different characteristics (e.g. image content or camera settings) from various sources to represent a real-world situation, which might be hardly available in practical forensic situations. Another drawback of all machine learning methods is that the assessment of their statistical performance still remains an open problem [18]. Hence, within this framework, the performance of the classifier is only evaluated empirically on a large image database and it is difficult to warrant a prescribed false alarm rate.

The approach proposed in this paper is based on hypothesis testing theory [19]. While the application of hypothesis testing is often more complex than the training of a classifier using machine learning methods, this first approach has indisputable advantages, see for instance [20] for discussion in steganalysis. Typically, this approach allows the design of a statistical test that is optimal with respect to a desired criterion, for instance minimizing false-alarm probability and maximizing detection power and, very often, permits the establishing of optimal test theoretical properties, that is the probabilities of false-alarm and miss detection. Besides, hypothesis testing usually provides valuable insight into the problem of how each parameter impact the performance of the optimal statistical test.

However, one of the main challenge to apply accurately hypothesis testing methods is that they requires an accurate statistical image model. In our prior works, hypothesis testing framework has already been exploited address the problem of camera model identification. More precisely, the first camera model identification method proposed within 
this framework has been targeting RAW images using heteroscedastic noise model [21, 23]. This noise model takes into account the contribution of Poisson noise in the RAW image acquisition process by characterizing the noise variance as a linear function of RAW pixel's expectation [24, 25]. However, the RAW format is hardly available in majority of practical forensics applications and most cameras output digital directly in JPEG format. Hence, for a more practical application, we have recently proposed an approach for camera model identification using Discrete Cosine Transform (DCT) coefficients from JPEG images [22, 26]. Those works exploited a state-of-the-art statistical model of DCT coefficients provided in $[27,28]$ that was obtained by studying and modeling the main steps involved in digital imaging of typical digital cameras [29].

It is important to note that the two main differences between the first approach proposed in $[21,23]$ and the latter one proposed in $[22,26]$ is that 1 ) the former exploits noise statistics in the spatial domain while the latter directly uses the DCT coefficients and 2) those approach are targeting different image format, RAW image for the first ones and JPEG compressed images for the latter ones.

It should be noted that, to the best of our knowledges, the problem camera device identification from rendered natural images (not RAW) in the spatial domain has not been studied within the framework of hypothesis testing theory. The main advantages of using pixel in spatial domain is that this information is always available regardless the file format and/or compression scheme. The goal of this paper is thus to study the design of an optimal detector, as referred to in [30], from rendered images and using pixel in spatial domain.

Recently, the study of noise statistics in the spatial domain of a rendered digital image has been performed in our previous research [31]. Since the heteroscedastic noise model characterizes accurately a RAW image, it is proposed to start from that model and take into account effects of post-acquisition processes to develop a so-called generalized signal-dependent noise model that has not been proposed yet in the literature. This noise model describes a non-linear relation between output pixel's expectation and variance. The generalized noise model can characterize an original rendered image format accurately, see more details in [31]. Similar to [21], the present paper exploits the generalized noise model to design a statistical test within hypothesis testing framework for camera model identification from JPEG images. The main contributions are the following:

- The approach is based on the generalized noise model that characterizes accurately the statistical properties of rendered digital images, after in-camera post-acquisition processes. Three parameters $(\tilde{a}, \tilde{b}, \gamma)$ that are specified in the generalized noise model are exploited as camera fingerprint for camera model identification.

- Stating the camera model identification problem in hypothesis testing framework, the paper proposes the optimal detector given by the Likelihood Ratio Test (LRT) in an ideal context where all model parameters are known. This optimal detector serves as an upper-bound of any statistical test for the camera model identification problem.

- In the practical context, the model parameters are unknown. The paper proposes two Generalized Likelihood Ratio Tests (GLRTs) to deal with the difficulty of unknown parameters. The statistical performance of the 


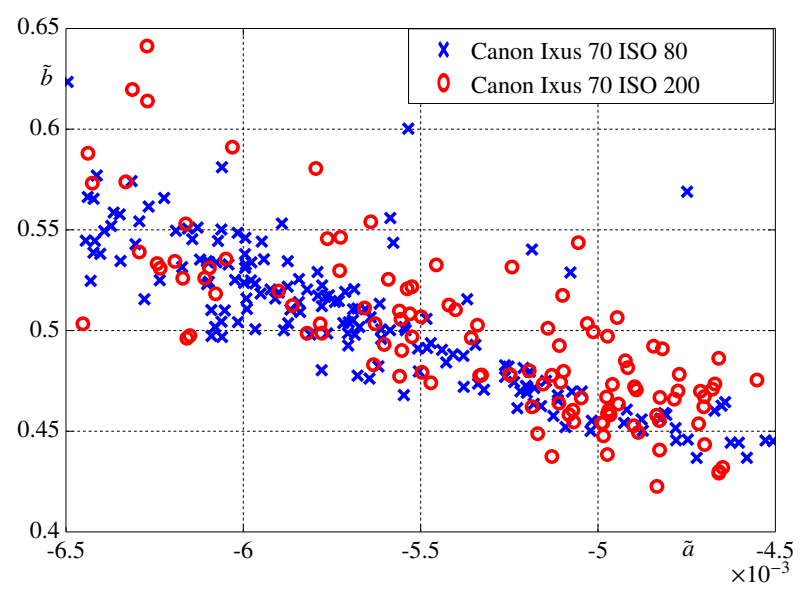

Figure 1: Estimated parameters ( $\tilde{a}, \tilde{b})$ on JPEG images issued from Canon Ixus 70 camera [32] with different camera settings.

GLRTs is analytically established. Moreover, the proposed GLRTs allow us to guarantee a prescribed falsealarm rate and the setting of decision threshold independently of the image content, which is crucial in an operational context. Numerical experiments also show that the loss of power of GLRTs compared with the LRT is negligible.

\subsection{Organization of the Paper}

The paper is organized as follows. Section 2 presents the generalized noise model and proposes the camera fingerprint that is further exploited for camera model identification. Section 3 states the camera model identification problem in the framework of hypothesis testing theory and studies the theoretical LRT assuming that all model parameters are known in advance. In practice, those parameters are unknown when inspecting a digital image. Section 4 designs two GLRTs to address the difficulty of unknown parameters. Section 5 presents numerical results of two proposed GLRTs on simulated and real natural JPEG images. Finally, Section 6 concludes the paper.

\section{Camera Fingerprint}

This section briefly describes main steps in the image processing pipeline of a digital camera and presents the generalized noise model that has been provided in [31]. Based on this noise model, a new camera fingerprint is proposed for camera model identification.

A typical image processing pipeline includes two stages: RAW image acquisition and post-acquisition processes (e.g. demosaicing, white-balancing and gamma correction), see more details in [4, 29, 27]. The output image is a full-color high-quality image, referred to as TIFF image. JPEG compression can also be performed for ease of storage and transmission.

The study of noise statistics in a natural image in TIFF or JPEG format has been accomplished in our previous work [31]. The RAW image can be characterized accurately by the heteroscedastic noise model since it takes into 


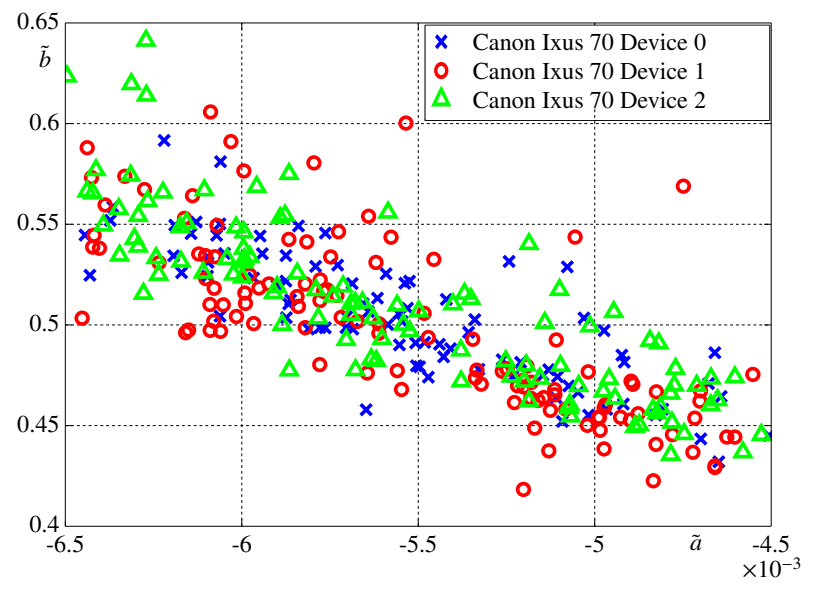

Figure 2: Estimated parameters $(\tilde{a}, \tilde{b})$ on JPEG images issued from different devices of Canon Ixus 70 model [32].

account the contribution of Poisson noise due to incident photons [21, 24, 25]. The heteroscedastic noise model is given as

$$
x_{i} \sim \mathcal{N}\left(\mu_{x_{i}}, a \mu_{x_{i}}+b\right)
$$

where $x_{i}$ denotes a RAW pixel and $\mu_{X}$ denotes the expectation of a random variable $X$. The parameters $(a, b)$ was proposed in our previous work [21] as fingerprint for camera model identification from RAW images. Then, it is proposed to start from the heteroscedastic noise model to study noise statistics in TIFF image. Assuming the operations of demosaicing and white-balancing are linear [4] and taking into account the non-linear effect of gamma correction, a so-called generalized noise model is given as

$$
\sigma_{z_{i}}^{2} \triangleq f\left(\mu_{z_{i}} ; \tilde{a}, \tilde{b}, \gamma\right)=\frac{1}{\gamma^{2}} \mu_{z_{i}}^{2-2 \gamma}\left(\tilde{a} \mu_{z_{i}}^{\gamma}+\tilde{b}\right)
$$

where $z_{i}$ denotes the output pixel, $\sigma_{X}^{2}$ denotes the variance of a random variable $X, \gamma$ is the correction factor (typically $\gamma=2.2)$, and $(\tilde{a}, \tilde{b})$ differ from the parameters $(a, b)$ due to the operations of demosaicing and white balancing. It is shown in [31] that the generalized noise model is also relevant to characterize JPEG images with moderate-tohigh quality factors $(Q F \geq 70)$. The model parameters $(\tilde{a}, \tilde{b}, \gamma)$ can be estimated by the Maximum Likelihood (ML) approach, see details in [31].

For camera model identification problem, it is necessary to evaluate the variability of the camera fingerprint for different camera settings and different devices per camera model, and to verify their discriminability for different camera models. Figure 1 shows the estimated parameters $(\tilde{a}, \tilde{b})$ on JPEG images of Canon Ixus 70 camera for different camera settings and Figure 2 shows the estimated parameters $(\tilde{a}, \tilde{b})$ on JPEG images acquired by different devices of Canon Ixus 70 model. Furthermore, Figure 3 illustrates the discriminability of the parameters for different camera models [31]. It is worth noting that the parameters $(\tilde{a}, \tilde{b})$ are invariant to imaged scenes and camera settings and discriminative for different camera models. Since the difference between estimated gamma factors of different camera models is small, we do not report them in this paper. The parameters $(\tilde{a}, \tilde{b}, \gamma)$ are relevant to be exploited as camera 


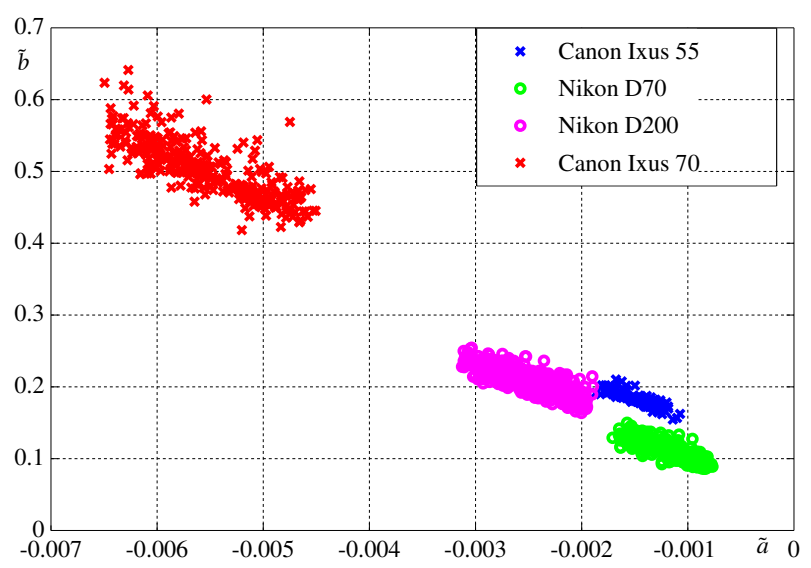

Figure 3: Estimated parameters $(\tilde{a}, \tilde{b})$ on JPEG images issued from different camera models, see [31] .

fingerprint to identify camera models.

\section{Optimal Detector for Camera Model Identification Problem}

The application of hypothesis testing theory requires to know the statistical distribution of a JPEG pixel. To this end, it is necessary to model the JPEG compression chain rigorously. JPEG compression mainly involves the Discrete Cosine Transform (DCT) and the quantization in the DCT domain, while the JPEG decompression performs dequantization and inverse DCT operation to return to spatial domain [33]. In general, the reconstructed image from a compressed file usually differs from the original image. There are two fundamental factors involved in this spatialdomain error [34]: the DCT basis vectors, and quantization error introduced in the DCT domain. The spatial-domain error at a pixel location is a weighted sum of 64 DCT-domain quantization errors within a $8 \times 8$ block. Providing an exact statistical distribution of the JPEG pixel is a challenging task due to the difficulty of establishing mathematically the model of DCT coefficients [29], characterizing the effect of quantization in the DCT domain [27, 28], and deriving the distribution of the sum of those random variables.

To overcome those difficulties, it is proposed to invoke the Lindeberg Central Limit Theorem (CLT) [19, theorem 11.2.5]. A preprocessing stage involves dividing the JPEG image $\mathbf{Z}$ into $K$ non-overlapping homogeneous segments $S_{k}$ of size $n_{k}, k \in\{1, \ldots, K\}$. In each segment $S_{k}$, the pixels $z_{k, i}, i \in\left\{1, \ldots, n_{k}\right\}$ are assumed to be independent and identically distributed. Such segmentation technique is detailed in [31]. In fact, this preprocessing stage is also performed for estimation of parameters $(\tilde{a}, \tilde{b}, \gamma)$. The JPEG pixel $z_{k, i}$ in the segment $S_{k}$ can be decomposed as

$$
z_{k, i}=\mu_{k}+\eta_{z k, i}
$$

where $\mu_{k}$ denotes the expectation of all pixels in the segment $S_{k}$ and $\eta_{z k, i}$ accounts for the spatial-domain noise after JPEG compression. Since the DCT can approximately decorrelate the input image [34], the spatial-domain noise $\eta_{z k, i}$ 


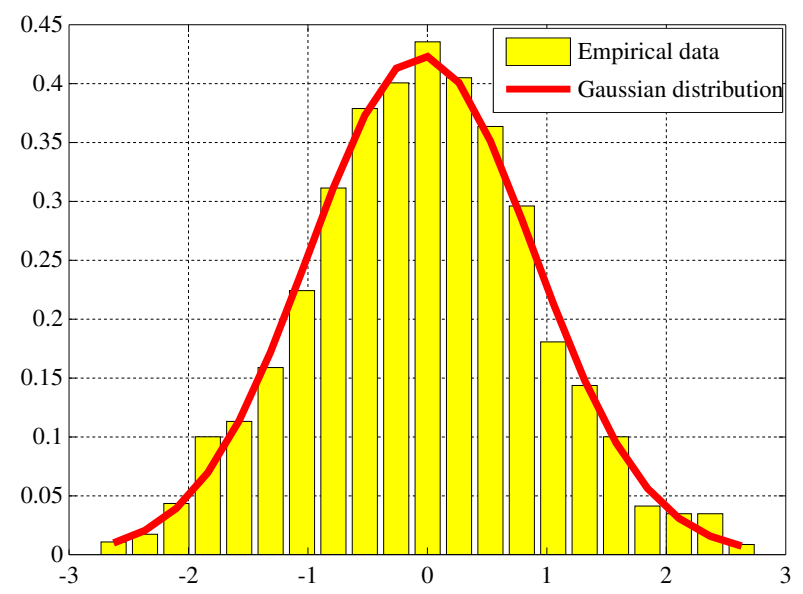

Figure 4: Empirical distribution of noise residuals in a segment of a natural JPEG image compared with theoretical Gaussian distribution.

in the decompressed JPEG image can be seen as a linear combination of independent random variables. In virtue of the Lindeberg CLT, the noise $\eta_{z_{k, i}}$ can be approximately modeled by the Gaussian distribution with zero-mean [34]. Meanwhile, the variance of noise $\eta_{z k, i}$ depends on pixel's expectation $\mu_{k}$ according to the generalized noise model (2). Figure 4 shows the empirical distribution of noise residuals in a segment extracted from a natural JPEG image, compared with theoretical Gaussian distribution. Therefore, it is proposed to model the JPEG pixel $z_{k, i}$ as

$$
z_{k, i} \sim \mathcal{N}\left(\mu_{k}, f\left(\mu_{k} ; \tilde{a}, \tilde{b}, \gamma\right)\right)
$$

\subsection{Hypothesis Testing Formulation}

Let analyze two camera models $\mathcal{S}_{0}$ and $\mathcal{S}_{1}$. Each camera model $\mathcal{S}_{j}, j \in\{0,1\}$ is characterized by three parameters $\left(\tilde{a}_{j}, \tilde{b}_{j}, \gamma_{j}\right)$. For obvious reasons, it is assumed that $\left(\tilde{a}_{0}, \tilde{b}_{0}, \gamma_{0}\right) \neq\left(\tilde{a}_{1}, \tilde{b}_{1}, \gamma_{1}\right)$. In a binary hypothesis testing, the inspected image $\mathbf{Z}$ is either acquired by camera model $\mathcal{S}_{0}$ or camera model $\mathcal{S}_{1}$. The goal of the test is to decide between two following hypotheses: $\forall k \in\{1, \ldots, K\}, \forall i \in\left\{1, \ldots, n_{k}\right\}$

$$
\left\{\begin{array}{l}
\mathcal{H}_{0}=\left\{z_{k, i} \sim \mathcal{N}\left(\mu_{k}, \sigma_{k, 0}^{2}\right)\right\} \\
\mathcal{H}_{1}=\left\{z_{k, i} \sim \mathcal{N}\left(\mu_{k}, \sigma_{k, 1}^{2}\right)\right\},
\end{array}\right.
$$

where $\sigma_{k, j}^{2}=f\left(\mu_{k} ; \tilde{a}_{j}, \tilde{b}_{j}, \gamma_{j}\right)$ is the noise variance with respect to the expectation $\mu_{k}$ under hypothesis $\mathcal{H}_{j}$. As previously explained, this paper focuses on designing a test that allows us to guarantee a prescribed false-alarm rate. Hence, let

$$
\mathcal{K}_{\alpha_{0}}=\left\{\delta: \sup _{\left(\mu, \tilde{a}_{0}, \tilde{b}_{0}, \gamma_{0}\right)} \mathbb{P}_{\mathcal{H}_{0}}\left[\delta(\mathbf{Z})=\mathcal{H}_{1}\right] \leq \alpha_{0}\right\}
$$

be the class of tests whose false alarm probability is upper-bounded by the rate $\alpha_{0}$. Here $\boldsymbol{\mu}=\left(\mu_{1}, \ldots, \mu_{K}\right)$ is the mean vector and $\mathbb{P}_{\mathcal{H}_{j}}[E]$ stands for the probability of event $E$ under hypothesis $\mathcal{H}_{j}, j \in\{0,1\}$, and the supremum over 
$\left(\boldsymbol{\mu}, \tilde{a}_{0}, \tilde{b}_{0}, \gamma_{0}\right)$ has to be understood as whatever model parameters might be. Among all the tests in the class $\mathcal{K}_{\alpha_{0}}$, it is aimed at finding a test $\delta$ which maximizes the power function $\beta_{\delta}$, defined by the correct detection probability:

$$
\beta_{\delta}=\mathbb{P}_{\mathcal{H}_{1}}\left[\delta(\mathbf{Z})=\mathcal{H}_{1}\right] .
$$

The problem (5) highlights three fundamental difficulties of the camera model identification. First, even when all model parameters $\left(\boldsymbol{\mu}, \tilde{a}_{j}, \tilde{b}_{j}, \gamma_{j}\right)$ are known, the most powerful test, namely the LRT, has never been studied in the literature. The second difficulty concerns unknown image parameters $\boldsymbol{\mu}$ in practice. Finally, the camera parameters $\left(\tilde{a}_{j}, \tilde{b}_{j}, \gamma_{j}\right)$ are also unknown, thus the hypothesis $\mathcal{H}_{j}$ becomes composite.

Suppose that the camera model $\mathcal{S}_{0}$ is available, thus forensic analysts can have access to its characteristics, or its fingerprints, i.e. its camera parameters $\left(\tilde{a}_{0}, \tilde{b}_{0}, \gamma_{0}\right)$ can be known. Therefore, they can make a decision by checking whether the image under investigation $\mathbf{Z}$ contains the fingerprint $\left(\tilde{a}_{0}, \tilde{b}_{0}, \gamma_{0}\right)$. In other words, it is proposed to solve the problem (5) when the alternative hypothesis $\mathcal{H}_{1}$ is composite, i.e. the camera parameters $\left(\tilde{a}_{1}, \tilde{b}_{1}, \gamma_{1}\right)$ are unknown. It can be noted that a test that maximizes the correct detection probability whatever $\left(\tilde{a}_{1}, \tilde{b}_{1}, \gamma_{1}\right)$ might scarcely exist. The main goal of this paper is to study the LRT and to design the GLRTs to address the second and third difficulties.

\subsection{Likelihood Ratio Test for Two Simple Hypotheses}

When all model parameters are known, in virtue of the Neyman-Pearson lemma [19, theorem 3.2.1], the most powerful test $\delta$ solving the problem (5) is the LRT given by the following decision rule

$$
\delta(\mathbf{Z})=\left\{\begin{array}{lll}
\mathcal{H}_{0} & \text { if } & \Lambda(\mathbf{Z})=\sum_{k=1}^{K} \sum_{i=1}^{n_{k}} \Lambda\left(z_{k, i}\right)<\tau \\
\mathcal{H}_{1} & \text { if } \quad \Lambda(\mathbf{Z})=\sum_{k=1}^{K} \sum_{i=1}^{n_{k}} \Lambda\left(z_{k, i}\right) \geq \tau,
\end{array}\right.
$$

where, to ensure that the LRT is in the class $\mathcal{K}_{\alpha_{0}}$, the decision threshold $\tau$ is the solution of the equation $\mathbb{P}_{\mathcal{H}_{0}}[\Lambda(\mathbf{Z}) \geq$ $\tau]=\alpha_{0}$ and the Likelihood Ratio (LR) of one observation $z_{k, i}$ is defined by

$$
\begin{aligned}
\Lambda\left(z_{k, i}\right) & =\log \frac{\frac{1}{\sqrt{2 \pi \sigma_{k, 1}^{2}}} \exp \left[-\frac{\left(z_{k, i}-\mu_{k}\right)^{2}}{2 \sigma_{k, 1}^{2}}\right]}{\frac{1}{\sqrt{2 \pi \sigma_{k, 0}^{2}}} \exp \left[-\frac{\left(z_{k, i}-\mu_{k}\right)^{2}}{2 \sigma_{k, 0}^{2}}\right]} \\
& =\frac{1}{2} \log \left(\frac{\sigma_{k, 0}^{2}}{\sigma_{k, 1}^{2}}\right)+\frac{1}{2}\left(\frac{1}{\sigma_{k, 0}^{2}}-\frac{1}{\sigma_{k, 1}^{2}}\right)\left(z_{k, i}-\mu_{k}\right)^{2} .
\end{aligned}
$$

In order to analytically establish the statistical performance of the LRT, it is necessary to characterize the statistical distribution of the LR $\Lambda(\mathbf{Z})$ under each hypothesis $\mathcal{H}_{j}$. To this end, the approach is based on the Lindeberg CLT [19, theorem 11.2.5] that requires to calculate the expectation and variance of $\Lambda\left(z_{k, i}\right)$. 
Proposition 1. Under hypothesis $\mathcal{H}_{j}$, the first two moments of the $L R \Lambda\left(z_{k, i}\right)$ is given by

$$
\begin{aligned}
& m_{k, j} \triangleq \mathbb{E}_{\mathcal{H}_{j}}\left[\Lambda\left(z_{k, i}\right)\right]=\frac{1}{2} \log \left(\frac{\sigma_{k, 0}^{2}}{\sigma_{k, 1}^{2}}\right)+\frac{1}{2}\left(\frac{1}{\sigma_{k, 0}^{2}}-\frac{1}{\sigma_{k, 1}^{2}}\right) \sigma_{k, j}^{2} \\
& v_{k, j} \triangleq \operatorname{Var}_{\mathcal{H}_{j}}\left[\Lambda\left(z_{k, i}\right)\right]=\frac{1}{2}\left(\frac{1}{\sigma_{k, 0}^{2}}-\frac{1}{\sigma_{k, 1}^{2}}\right)^{2} \sigma_{k, j}^{4},
\end{aligned}
$$

where $\mathbb{E}_{\mathcal{H}_{j}}[\cdot]$ and $\operatorname{Var}_{\mathcal{H}_{j}}[\cdot]$ denote respectively the mathematical expectation and variance under hypothesis $\mathcal{H}_{j}$.

Proof. The proof of Proposition 1 is given in Appendix A.

In virtue of Lindeberg CLT, the statistical distribution of the $\operatorname{LR} \Lambda(\mathbf{Z})$ under hypothesis $\mathcal{H}_{j}$ is derived as

$$
\Lambda(\mathbf{Z}) \stackrel{D}{\longrightarrow} \mathcal{N}\left(m_{j}, v_{j}\right)
$$

where the notation $\stackrel{D}{\longrightarrow}$ denotes the convergence in distribution and

$$
\begin{gathered}
m_{j}=\sum_{k=1}^{K} \sum_{i=1}^{n_{k}} m_{k, j}=\sum_{k=1}^{K} n_{k} m_{k, j} \\
v_{j}=\sum_{k=1}^{K} \sum_{i=1}^{n_{k}} v_{k, j}=\sum_{k=1}^{K} n_{k} v_{k, j} .
\end{gathered}
$$

Since a natural image is heterogeneous, it is proposed to normalize the $\operatorname{LR} \Lambda(\mathbf{Z})$ in order to set the decision threshold independently of the image content. The normalized LR is defined by

$$
\Lambda^{\star}(\mathbf{Z})=\frac{\Lambda(\mathbf{Z})-m_{0}}{\sqrt{v_{0}}} .
$$

The $\operatorname{LR} \Lambda(\mathbf{Z})$ differs from the normalized $\operatorname{LR} \Lambda^{\star}(\mathbf{Z})$ only by an additive constant and a multiplicative constant, which does not change the decision rule given by the LRT. Accordingly, the corresponding test $\delta^{\star}$ is rewritten as follows

$$
\delta^{\star}(\mathbf{Z})=\left\{\begin{array}{lll}
\mathcal{H}_{0} & \text { if } & \Lambda^{\star}(\mathbf{Z})<\tau^{\star} \\
\mathcal{H}_{1} & \text { if } & \Lambda^{\star}(\mathbf{Z}) \geq \tau^{\star},
\end{array}\right.
$$

where the decision threshold $\tau^{\star}$ is the solution of the equation $\mathbb{P}_{\mathcal{H}_{0}}\left[\Lambda^{\star}(\mathbf{Z}) \geq \tau^{\star}\right]=\alpha_{0}$. The decision threshold $\tau^{\star}$ and the power function $\beta_{\delta^{\star}}$ are given in the following theorem:

Theorem 1. In an ideal context where all the model parameters $\left(\boldsymbol{\mu}, \tilde{a}_{j}, \tilde{b}_{j}, \gamma\right)$ are known in advance, the decision threshold and the power function of the LRT $\delta^{\star}$ are given by

$$
\begin{aligned}
\tau^{\star} & =\Phi^{-1}\left(1-\alpha_{0}\right) \\
\beta_{\delta^{\star}} & =1-\Phi\left(\frac{m_{0}-m_{1}+\tau^{\star} \sqrt{v_{0}}}{\sqrt{v_{1}}}\right),
\end{aligned}
$$

where $\Phi(\cdot)$ and $\Phi^{-1}(\cdot)$ denotes respectively the cumulative distribution function of the standard Gaussian random variable and its inverse. 
Proof. For the sake of clarity, the proof of Theorem 1 is given in Appendix B.

From Theorem 1, it can be noted that the decision threshold $\tau^{\star}$ is now independent of the image content. Therefore the LRT $\delta^{\star}$ can be applied to any natural image. The LRT $\delta^{\star}$ allows us to warrant a prescribed false alarm rate and maximizes the correct detection probability. Since its statistical performance is analytically established, it can provide an analytically predictable result for any false alarm rate $\alpha_{0}$. The detection power $\beta_{\delta^{\star}}$ serves as an upper-bound of any statistical test for the camera model identification problem.

\section{Practical Context: Generalized Likelihood Ratio Test}

The scenario studied in the LRT may not be realistic because the parameters $\left(\mu_{k}, \tilde{a}_{1}, \tilde{b}_{1}, \gamma_{1}\right)$ are unknown in practice. This section designs two GLRTs to deal with the difficulty of unknown parameters. It is proposed to replace unknown parameters by their ML estimates in the $\operatorname{LR} \Lambda\left(z_{k, i}\right)(8)$.

\subsection{Generalized Likelihood Ratio Test with Unknown Image Parameters}

The GLRT designed in this subsection deals with the difficulty of unknown image parameters $\mu_{k}$ assuming that the camera parameters $\left(\tilde{a}_{0}, \tilde{b}_{0}, \gamma_{0}\right)$ and $\left(\tilde{a}_{1}, \tilde{b}_{1}, \gamma_{1}\right)$ are known, i.e. the inspected image $\mathbf{Z}$ is either acquired by the known camera model $\mathcal{S}_{0}$ or the known camera model $\mathcal{S}_{1}$.

By replacing the unknown parameter $\mu_{k}$ by its estimate $\hat{\mu}_{k}$ in the $\operatorname{LR} \Lambda\left(z_{k, i}\right)(8)$, the Generalized Likelihood Ratio (GLR) $\widehat{\Lambda}_{1}\left(z_{k, i}\right)$ is given by

$$
\widehat{\Lambda}_{1}\left(z_{k, i}\right)=\frac{1}{2} \log \left(\frac{\hat{\sigma}_{k, 0}^{2}}{\hat{\sigma}_{k, 1}^{2}}\right)+\frac{1}{2}\left(\frac{1}{\hat{\sigma}_{k, 0}^{2}}-\frac{1}{\hat{\sigma}_{k, 1}^{2}}\right)\left(z_{k, i}-\hat{\mu}_{k}\right)^{2},
$$

where $\hat{\sigma}_{k, j}^{2}=f\left(\hat{\mu}_{k} ; \tilde{a}_{j}, \tilde{b}_{j}, \gamma_{j}\right)$. In the estimation method proposed in [31], it is assumed that the variance of the estimates $\hat{\mu}_{k}$ is negligible when the number of pixels is large. Therefore, the mathematical expectation and variance of the GLR $\widehat{\Lambda}_{1}\left(z_{k, i}\right)$ do not change. Consequently, the statistical distribution of the GLR $\widehat{\Lambda}_{1}(\mathbf{Z})=\sum_{k=1}^{K} \sum_{i=1}^{n_{k}} \widehat{\Lambda}_{1}\left(z_{k, i}\right)$ under hypothesis $\mathcal{H}_{j}$ can be approximated as

$$
\widehat{\Lambda}_{1}(\mathbf{Z}) \stackrel{D}{\longrightarrow} \mathcal{N}\left(m_{j}, v_{j}\right)
$$

where the expectation $m_{j}$ and $v_{j}$ are given respectively in (12) and (13).

Similarly, the normalized GLR $\widehat{\Lambda}_{1}^{\star}(\mathbf{Z})$ can be defined as $\widehat{\Lambda}_{1}^{\star}(\mathbf{Z})=\frac{\widehat{\Lambda}_{1}(\mathbf{Z})-m_{0}}{\sqrt{v_{0}}}$. However, the expectation $m_{0}$ and variance $v_{0}$ can not be defined in practice since the parameters $\mu_{k}$ are unknown. Therefore, it is proposed to replace $\mu_{k}$ by $\hat{\mu}_{k}$ in (12) and (13) to obtain estimates of $m_{0}$ and $v_{0}$, denoted $\hat{m}_{0}$ and $\hat{v}_{0}$. The normalized GLR $\widehat{\Lambda}_{1}^{\star}(\mathbf{Z})$ is given in practice as

$$
\widehat{\Lambda}_{1}^{\star}(\mathbf{Z})=\frac{\widehat{\Lambda}_{1}(\mathbf{Z})-\hat{m}_{0}}{\sqrt{\hat{v}_{0}}} .
$$


Since the variance of the estimates $\hat{m}_{0}$ and $\hat{v}_{0}$ is negligible, it follows that

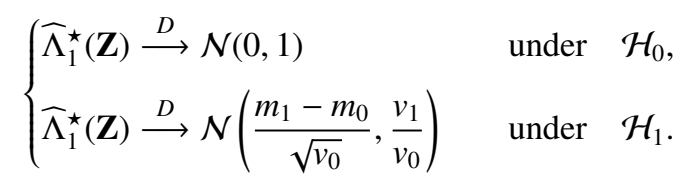

Finally, the GLRT $\widehat{\delta}_{1}^{\star}$ based on the normalized GLR $\widehat{\Lambda}_{1}^{\star}(\mathbf{Z})$ is given by

$$
\widehat{\delta}_{1}^{\star}(\mathbf{Z})=\left\{\begin{array}{lll}
\mathcal{H}_{0} & \text { if } & \widehat{\Lambda}_{1}^{\star}(\mathbf{Z})<\widehat{\tau}_{1}^{\star} \\
\mathcal{H}_{1} & \text { if } & \widehat{\Lambda}_{1}^{\star}(\mathbf{Z}) \geq \widehat{\tau}_{1}^{\star},
\end{array}\right.
$$

where the decision threshold $\widehat{\tau}_{1}^{\star}$ is the solution of the equation $\mathbb{P}_{\mathcal{H}_{0}}\left[\widehat{\Lambda}_{1}^{\star}(\mathbf{Z}) \geq \widehat{\tau}_{1}^{\star}\right]=\alpha_{0}$. From $(21)$, the decision threshold and the power of the GLRT $\widehat{\delta}_{1}^{\star}$ can be accordingly defined as in the Theorem 1 .

\subsection{Generalized Likelihood Ratio Test with Unknown Image and Camera Parameters}

This subsection deals with a scenario where the image parameters $\mu_{k}$ and the camera parameters $\left(\tilde{a}_{1}, \tilde{b}_{1}, \gamma\right)$ are unknown. Thus the hypothesis $\mathcal{H}_{1}$ becomes composite. The GLRT designed in this subsection aims to verify whether the inspected image $\mathbf{Z}$ is acquired by the camera model $\mathcal{S}_{0}$. The inspected image $\mathbf{Z}$ is allowed to be taken from an unknown camera model.

Before designing the GLRT, the ML estimation of camera parameters $\left(\tilde{a}_{1}, \tilde{b}_{1}, \gamma_{1}\right)$ is performed on the inspected image $\mathbf{Z}$; see details in [31]. Here we set $\gamma_{1}=\gamma_{0}$ and the estimation problem with three parameters is reduced to the one with two parameters $\left(\tilde{a}_{1}, \tilde{b}_{1}\right)$. The ML estimates $\left(\hat{\tilde{a}}_{1}, \hat{\tilde{b}}_{1}\right)$ are asymptotically consistent [19], i.e. they asymptotically converge in probability to their true value: $\hat{\tilde{a}}_{1} \stackrel{P}{\longrightarrow} \tilde{a}_{1}$ and $\hat{\tilde{b}}_{1} \stackrel{P}{\longrightarrow} \tilde{b}_{1}$. The parameters $\left(\tilde{a}_{1}, \tilde{b}_{1}, \gamma_{1}\right)$ would characterize a certains unknown camera model. Furthermore, the ML estimates $\left(\hat{\tilde{a}}_{1}, \hat{\tilde{b}}_{1}\right)$ exhibit a certain variability. Let $\sigma_{\tilde{a}_{1}}^{2}, \sigma_{\tilde{b}_{1}}^{2}, \sigma_{\tilde{a}_{1} \tilde{b}_{1}}$ denote respectively the variance of $\hat{\tilde{a}}_{1}$, the variance of $\hat{\tilde{b}}_{1}$ and the covariance between $\hat{\tilde{a}}_{1}$ and $\hat{\tilde{b}}_{1}$.

By replacing $\left(\mu_{k}, \tilde{a}_{1}, \tilde{b}_{1}\right)$ by $\left(\hat{\mu}_{k}, \hat{\tilde{a}}_{1}, \hat{\tilde{b}}_{1}\right)$ in $(8)$, the GLR $\widehat{\Lambda}_{2}\left(z_{k, i}\right)$ is now given by

$$
\begin{aligned}
& \widehat{\Lambda}_{2}\left(z_{k, i}\right)=\frac{1}{2} \log \frac{f\left(\hat{\mu}_{k} ; \tilde{a}_{0}, \tilde{b}_{0}, \gamma_{0}\right)}{f\left(\hat{\mu}_{k} ; \hat{\tilde{a}}_{1}, \hat{\tilde{b}}_{1}, \gamma_{1}\right)} \\
& +\frac{f\left(\hat{\mu}_{k} ; \hat{\tilde{a}}_{1}, \hat{\tilde{b}}_{1}, \gamma_{1}\right)-f\left(\hat{\mu}_{k} ; \tilde{a}_{0}, \tilde{b}_{0}, \gamma_{0}\right)}{2 f\left(\hat{\mu}_{k} ; \hat{\tilde{a}}_{1}, \hat{\tilde{b}}_{1}, \gamma_{1}\right) f\left(\hat{\mu}_{k} ; \tilde{a}_{0}, \tilde{b}_{0}, \gamma_{0}\right)}\left(z_{k, i}-\hat{\mu}_{k}\right)^{2} .
\end{aligned}
$$

Proposition 2. Under hypothesis $\mathcal{H}_{j}$, using the Delta method [19, theorem 11.2.14], the first two moments of the GLR $\widehat{\Lambda}_{2}\left(z_{k, i}\right)$ can be approximated as

$$
\begin{aligned}
\mathbb{E}_{\mathcal{H}_{j}}\left[\widehat{\Lambda}_{2}\left(z_{k, i}\right)\right]= & m_{k, j} \\
\operatorname{Var}_{\mathcal{H}_{j}}\left[\widehat{\Lambda}_{2}\left(z_{k, i}\right)\right]= & v_{k, j}+\frac{1}{4} \frac{\operatorname{Var}_{\mathcal{H}_{j}}\left[f\left(\hat{\mu}_{k} ; \hat{\tilde{a}}_{1}, \hat{\tilde{b}}_{1}, \gamma_{1}\right)\right]}{\sigma_{k, 1}^{4}} \\
& +\frac{3}{4} \frac{\operatorname{Var}_{\mathcal{H}_{j}}\left[f\left(\hat{\mu}_{k} ; \hat{\tilde{a}}_{1}, \hat{\tilde{b}}_{1}, \gamma_{1}\right)\right]}{\sigma_{k, 1}^{8}} \sigma_{k, j}^{4},
\end{aligned}
$$


where $\operatorname{Var}_{\mathcal{H}_{j}}\left[f\left(\hat{\mu}_{k} ; \hat{\tilde{a}}_{1}, \hat{\tilde{b}}_{1}, \gamma_{1}\right)\right]$ is given by

$$
\begin{aligned}
\operatorname{Var}_{\mathcal{H}_{j}}\left[f\left(\hat{\mu}_{k} ; \hat{\tilde{a}}_{1}, \hat{\tilde{b}}_{1}, \gamma_{1}\right)\right]= & \frac{\mu_{k}^{4-2 \gamma_{1}}}{\gamma_{1}^{4}} \sigma_{\tilde{a}_{1}}^{2}+\frac{\mu_{k}^{4-4 \gamma_{1}}}{\gamma_{1}^{4}} \sigma_{\tilde{b}_{1}}^{2} \\
& +2 \frac{\mu_{k}^{4-3 \gamma_{1}}}{\gamma_{1}^{4}} \sigma_{\tilde{a}_{1} \tilde{b}_{1}} .
\end{aligned}
$$

Proof. The proof of Proposition 2 is given in Appendix C.

It can be noted that the mathematical expectation of the GLR $\widehat{\Lambda}_{2}\left(z_{k, i}\right)$ does not change. Besides, the second and the last terms in (25) aim to take into account the variability of $\left(\hat{\tilde{a}}_{1}, \hat{\tilde{b}}_{1}\right)$ in the variance of the GLR $\widehat{\Lambda}_{2}\left(z_{k, i}\right)$. For brevity, let denote $\tilde{v}_{k, j}=\operatorname{Var}_{\mathcal{H}_{j}}\left[\widehat{\Lambda}_{2}\left(z_{k, i}\right)\right]$. In virtue of the Lindeberg CLT, the GLR $\widehat{\Lambda}_{2}(\mathbf{Z})=\sum_{k=1}^{K} \sum_{i=1}^{n_{k}} \widehat{\Lambda}_{2}\left(z_{k, i}\right)$ follows the Gaussian distribution under hypothesis $\mathcal{H}_{j}$

$$
\widehat{\Lambda}_{2}(\mathbf{Z}) \stackrel{D}{\longrightarrow} \mathcal{N}\left(m_{j}, \tilde{v}_{j}\right) .
$$

where the expectation $m_{j}$ is given in (12) and the variance $\tilde{v}_{j}=\sum_{k=1}^{K} n_{k} \tilde{v}_{k, j}$.

Finally, the GLRT $\widehat{\delta}_{2}^{\star}$ based on the normalized GLR $\widehat{\Lambda}_{2}^{\star}(\mathbf{Z})=\frac{\widehat{\Lambda}_{2}(\mathbf{Z})-\hat{m}_{0}}{\sqrt{\hat{v}_{0}}}$ is written as

$$
\widehat{\delta}_{2}^{\star}(\mathbf{Z})=\left\{\begin{array}{lll}
\mathcal{H}_{0} & \text { if } & \widehat{\Lambda}_{2}^{\star}(\mathbf{Z})<\widehat{\tau}_{2}^{\star} \\
\mathcal{H}_{1} & \text { if } & \widehat{\Lambda}_{2}^{\star}(\mathbf{Z}) \geq \widehat{\tau}_{2}^{\star},
\end{array}\right.
$$

where $\hat{m}_{0}$ and $\hat{\tilde{v}}_{0}$ are estimates of $m_{0}$ and $\tilde{v}_{0}$ by replacing unknown parameters $\left(\mu_{k}, \tilde{a}_{1}, \tilde{b}_{1}\right)$ by $\left(\hat{\mu}_{k}, \hat{\tilde{a}}_{1}, \hat{\vec{b}}_{1}\right)$, and the decision threshold $\widehat{\tau}_{2}^{\star}$ is the solution of the equation $\mathbb{P}_{\mathcal{H}_{0}}\left[\widehat{\Lambda}_{2}^{\star}(\mathbf{Z}) \geq \widehat{\tau}_{2}^{\star}\right]=\alpha_{0}$.

Theorem 2. When the image $\mathbf{Z}$ is tested against the known camera model $\mathcal{S}_{0}$ characterized by $\left(\tilde{a}_{0}, \tilde{b}_{0}, \gamma_{0}\right)$, the decision threshold and the power function of the GLRT $\widehat{\delta}_{2}^{\star}$ are given by

$$
\begin{aligned}
& \widehat{\tau}_{2}^{\star}=\Phi^{-1}\left(1-\alpha_{0}\right) \\
& \beta_{\widetilde{\delta}_{2}^{\star}}=1-\Phi\left(\frac{m_{0}-m_{1}+\widehat{\tau}_{2}^{\star} \sqrt{\tilde{v}_{0}}}{\sqrt{\tilde{v}_{1}}}\right) .
\end{aligned}
$$

Proof. The proof of Theorem 2 is given in Appendix D.

The statistical performance of the proposed GLRTs $\widehat{\delta}_{1}^{\star}$ and $\widehat{\delta}_{2}^{\star}$ is analytically provided. Moreover, they allow us to warrant a prescribed false alarm rate and set the decision threshold independently of image content (see (16) and (29)). It is worth noting that the GLRT $\widehat{\delta}_{1}^{\star}$ can be interpreted as a closed hypothesis testing since the decision is made only between two known camera models $\mathcal{S}_{0}$ and $\mathcal{S}_{1}$. Meanwhile, the GLRT $\widehat{\delta}_{2}^{\star}$ becomes an open hypothesis testing because it aims to verify whether the given image is acquired by camera model $\mathcal{S}_{0}$ or not. These two proposed GLRTs can be straightforwardly applied in practice, depending on the requirements of the operational context. 


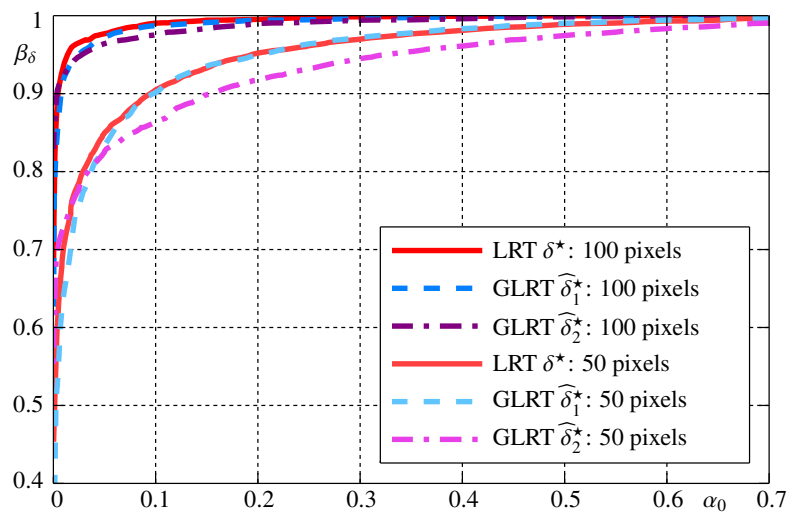

Figure 5: Detection performance of the proposed tests for 50 and 100 pixels extracted randomly from simulated JPEG images with quality factor 100

\section{Numerical Experiments}

\subsection{Receiver Operating Characteristic Curve on Simulated Database}

The implementation of the GLRT $\widehat{\delta}_{2}^{\star}$ requires to know the covariance matrix of ML estimates $\left(\hat{\tilde{a}}_{1}, \hat{\tilde{b}}_{1}\right)$. However, the ML estimates $\left(\hat{\tilde{a}}_{1}, \hat{\tilde{b}}_{1}\right)$ are solved numerically [31], which causes a difficulty of defining their statistical properties. To overcome this difficulty, firstly it is proposed to estimate the parameters $(\tilde{a}, \tilde{b}, \gamma)$ on each image from 50 images taken by the camera model $\mathcal{S}_{0}$ since this camera model is assumed to be available. These images are selected randomly to cover different image contents. Then the averaged gamma is calculated over 50 previous gamma values. To reduce the variability of the estimates, we set the parameter $\gamma$ to the averaged gamma value and re-estimate the parameters $(\tilde{a}, \tilde{b})$ on each image. Consequently, the empirical covariance matrix can be calculated from 50 couples $(\hat{\tilde{a}}, \hat{\tilde{b}})$. This empirical covariance matrix is used for the GLRT $\widehat{\delta}_{2}^{\star}$. Strictly speaking, this covariance matrix characterizes the variability of the camera parameters $\left(\tilde{a}_{0}, \tilde{b}_{0}\right)$. By doing so, it is expected that the estimates $\left(\hat{\tilde{a}}_{1}, \hat{\tilde{b}}_{1}\right)$ would fall into the neighborhood of the camera parameters $\left(\tilde{a}_{0}, \tilde{b}_{0}\right)$. In other words, the inspected image $\mathbf{Z}$ is expected to be taken by the camera model $\mathcal{S}_{0}$. This step is also performed in the test with real images.

The detection performance of the proposed tests is first theoretically studied on a simulated database. Suppose the camera model $\mathcal{S}_{0}$ and $\mathcal{S}_{1}$ are respectively characterized by $\left(\tilde{a}_{0}, \tilde{b}_{0}, \gamma_{0}\right)=(-0.0012,0.11,0.8)$ and $\left(\tilde{a}_{1}, \tilde{b}_{1}, \gamma_{1}\right)=$ $(-0.0025,0.20,0.85)$. These parameters respectively correspond to Nikon D70 and Nikon D200 camera models in the Dresden image database [32], see Figure 3 and also details in [31]. From a simple synthetic image given in [25], those camera parameters are used with the generalized noise model (2) to generate randomly 5000 images for camera model $\mathcal{S}_{0}$ and 5000 images for camera model $\mathcal{S}_{1}$. These images are further compressed with different quality factors using the software imagemagick. Therefore, these images could follow the image processing pipeline as described in Section 2.

Firstly, it is desirable to compare the detection performance of the practical GLRTs with the theoretical LRT 


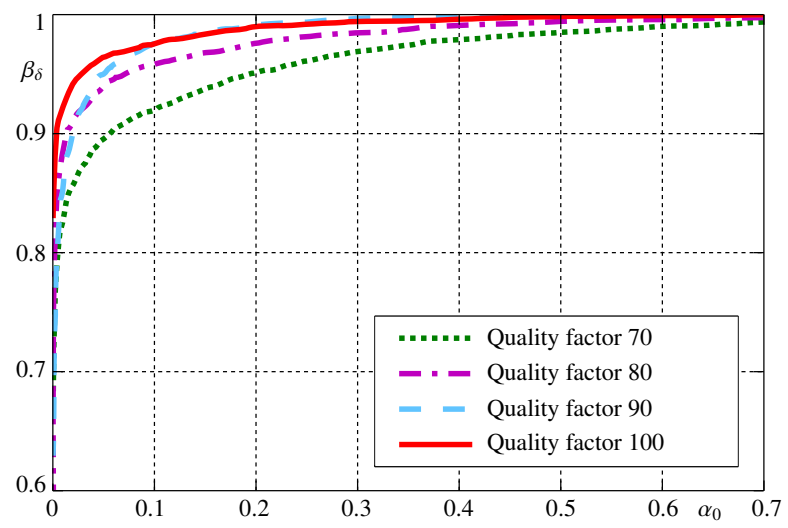

Figure 6: Detection performance of the GLRT $\widehat{\delta}_{2}^{\star}$ for 100 pixels extracted randomly from simulated JPEG images with different quality factors.

to observe the loss of power in the GLRTs caused by the estimation error of unknown parameters. The detection performance of the proposed tests for different number of pixels is illustrated in Figure 5. The pixels in the tests are extracted randomly. The power function $\beta_{\delta}$ of each test is plotted as a function of the false alarm rate $\alpha_{0}$. It can be noted that the loss of power between the LRT $\delta^{\star}$ and the GLRT $\widehat{\delta}_{1}^{\star}$ is negligible even with a small number of pixels (e.g. 50 pixels), which also indicates that the parameters $\mu_{k}$ are well estimated. Moreover, from Figure 5, a small loss of power between the GLRT $\widehat{\delta}_{1}^{\star}$ and the GLRT $\widehat{\delta}_{2}^{\star}$ is revealed. Nevertheless, this loss of power decreases when the number of pixels increases. Actually, the fact of selecting randomly a number of pixels (e.g. 50 and 100 pixels) for the proposed tests allows a better visibility since their power function is perfect (i.e. $\beta_{\delta}=1$ ) from only 500 pixels for any false alarm rate. On the contrary to other methods that exploit all the pixels, only a small number of pixels is required to achieve a high detection performance, which emphasizes the strength of the proposed approach. This observation could be useful in case of real images.

Furthermore, it is necessary to study the impact of JPEG compression factor on the detection performance of the GLRT $\widehat{\delta}_{2}^{\star}$. As mentioned in Section 2, the generalized noise model is also relevant for JPEG images with moderateto-high quality factors $(Q F \geq 70)$. Figure 6 shows its detection performance for different quality factors $(Q F=$ $70,80,90,100)$. As expected, the correct detection probability $\beta_{\delta}$ decreases with the decline of the quality factor since the compression error intervenes more important in the generalized noise model.

\subsection{Receiver Operating Characteristic Curve on Two Nikon D70 and Nikon D200 Cameras}

In hypothesis testing framework studied in this paper, the camera fingerprint $\left(\tilde{a}_{0}, \tilde{b}_{0}, \gamma_{0}\right)$ is assumed to be known in advance. Therefore, this fingerprint needs to be defined accurately in practice. To this end, it is proposed to estimate the parameters $\left(\tilde{a}_{0}, \tilde{b}_{0}, \gamma_{0}\right)$ using the same technique of calculating the covariance matrix proposed in Section 5.1 . Firstly, the estimates $(\hat{a}, \hat{\tilde{b}}, \hat{\gamma})$ on each image are given and the reference parameter $\gamma_{0}$ is calculated as the average of 50 gamma values. Then the parameters $(\tilde{a}, \tilde{b})$ are re-estimated on each image by setting $\gamma$ to the reference $\gamma_{0}$. The reference parameters $\left(\tilde{a}_{0}, \tilde{b}_{0}\right)$ are finally obtained by averaging the previous estimates $(\hat{\tilde{a}}, \hat{\tilde{b}})$. The covariance matrix of 


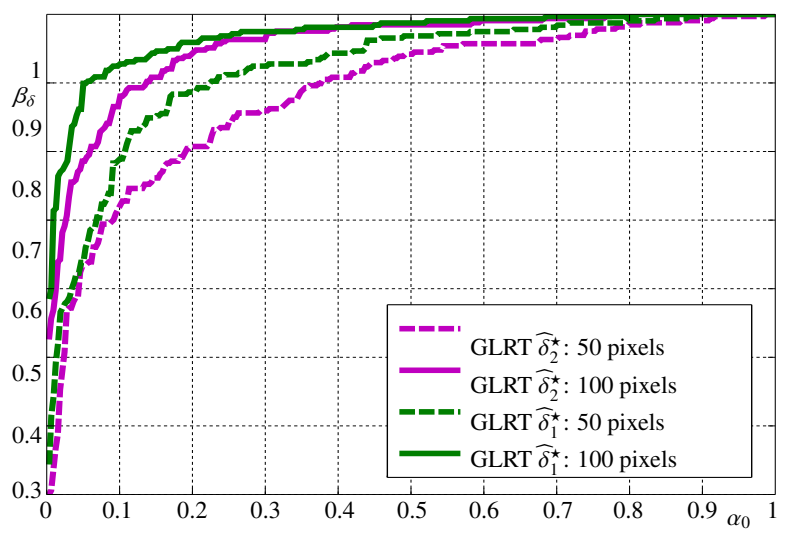

Figure 7: Detection performance of the GLRT $\widehat{\delta}_{1}^{\star}$ and $\widehat{\delta}_{2}^{\star}$ for 50 and 100 pixels extracted randomly from JPEG images of Nikon D70 and Nikon D200 cameras.

$\left(\tilde{a}_{0}, \tilde{b}_{0}\right)$ can be also defined. Evidently, using more images will get a better estimate but it is also less realistic. The number of 50 is a good trade-off.

To highlight the relevance of the proposed GLRTs, two Nikon D70 and Nikon D200 camera models of the Dresden image database [32] are chosen to conduct experiments since two camera models of the same brand are expected to exhibit similar characteristics. Only the red color channel is used in this experiment. The Nikon D70 and Nikon D200 cameras are respectively set at $\mathcal{H}_{0}$ and $\mathcal{H}_{1}$. The reference parameters of each camera model are defined as discussed above. Figure 7 shows the detection performance of the GLRTs $\widehat{\delta}_{1}^{\star}$ and $\widehat{\delta}_{2}^{\star}$ for different numbers of pixels. We can note a similar behavior to the detection performance on the simulated database. There is a small loss of power between the two power functions since the estimates $\left(\hat{\tilde{a}}_{1}, \hat{\tilde{b}}_{1}\right)$ used in the GLRT $\widehat{\delta}_{2}^{\star}$ are influenced by the image content. Nevertheless, this loss of power also decreases as the number of pixels increases. Two proposed GLRTs are nearly perfect from 500 pixels.

Furthermore, as mentioned above, the proposed tests allow us to warrant a prescribed false alarm probability. Therefore, it is desirable to compare the empirical false alarm probability of the proposed GLRTs in practice with theoretical one given in (16) and (29). Figure 8 shows the comparison between the theoretical and empirical false alarm probability, which are plotted as a function of decision threshold $\tau$. The two proposed GLRTs $\widehat{\delta}_{1}^{\star}$ and $\widehat{\delta}_{2}^{\star}$ show an ability to guarantee a prescribed false alarm rate, even though there is a slight difference in some cases (typically $\alpha_{0} \leq 10^{-3}$ ) due to the influence of image content, the presence of weak outliers in the segments $S_{k}$, and the inaccuracy of the CLT for modeling tails.

\subsection{Results on a Large Database}

Experiments are then conducted on a large database to verify the efficiency of the proposed approach. The public Dresden image database [32] is chosen in our experiments. Technical specifications of the cameras are shown in Table 1, see more details in [32]. The database covers different devices per camera model, different imaged scenes, different camera settings and different environmental conditions. The images are acquired with different JPEG quality factors. 


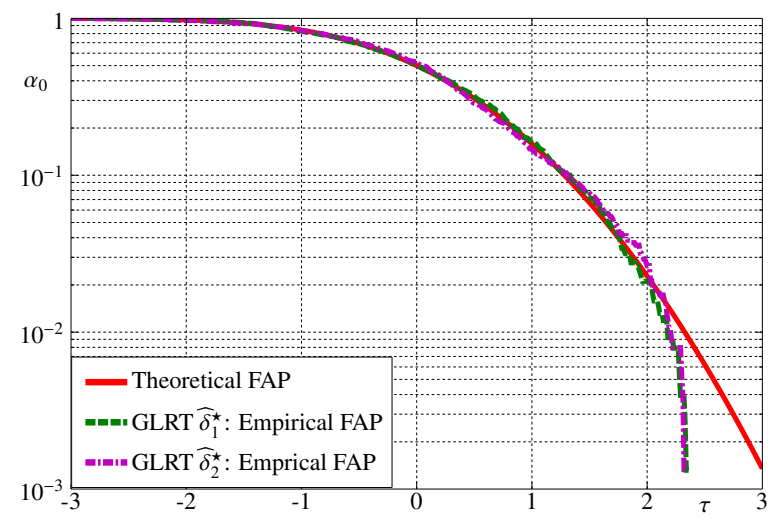

Figure 8: Comparison between the theoretical false alarm probability (FAP) and the empirical FAP, plotted as a function of decision threshold $\tau$.

For each camera model, 50 images are used to estimate the reference parameters and other images are used for the testing stage.

Firstly, the GLRT $\widehat{\delta}_{2}^{\star}$ is conducted to verify whether a given image is acquired by the camera model of interest. The decision threshold $\widehat{\tau}_{2}^{\star}$ is given by the Theorem 2 corresponding to the false alarm rate $\alpha_{0}=10^{-5}$. If the normalized GLR $\widehat{\Lambda}_{2}^{\star}(\mathbf{Z})$ is smaller than the decision threshold $\widehat{\tau}_{2}^{\star}$, the hypothesis $\mathcal{H}_{0}$ is accepted, i.e. the given image is declared taken by the camera model of interest. On the contrary, the hypothesis $\mathcal{H}_{1}$ is accepted. The detection performance of the GLRT $\widehat{\delta}_{2}^{\star}$ is shown in Table 2 . In this table, each camera model is considered as hypothesis $\mathcal{H}_{0}$ (row) and all images (column) are tested against $\mathcal{H}_{0}$. The values in the table 2 indicate the percentage of images that are detected taken by the camera model $\mathcal{H}_{0}$. The table in this paper is not used in the same way as in the classification in which the sum for each class yields $100 \%$. The inspected image is brought into the binary testing of the known camera model

\begin{tabular}{lc|ccc}
\hline \hline Camera Model & & No. devices & Resolution & No. images \\
\hline Canon Ixus 70 & $\mathrm{Cn}$ & 3 & $3072 \times 2304$ & 500 \\
Casio EX-Z150 & $\mathrm{Cs}$ & 5 & $3264 \times 2448$ & 600 \\
Fujifilm J50 & $\mathrm{F}$ & 3 & $3264 \times 2448$ & 500 \\
Nikon D200 & $\mathrm{N} 1$ & 2 & $3872 \times 2592$ & 500 \\
Nikon D70 & $\mathrm{N} 2$ & 2 & $3008 \times 2000$ & 300 \\
Olympus 1050SW & $\mathrm{O}$ & 5 & $3648 \times 2736$ & 700 \\
Panasonic FZ50 & $\mathrm{Pa}$ & 3 & $3648 \times 2736$ & 600 \\
Pentax A40 & $\mathrm{Pe}$ & 4 & $4000 \times 3000$ & 600 \\
Praktica DCZ & $\mathrm{Pr}$ & 5 & $2560 \times 1920$ & 700 \\
Ricoh GX100 & $\mathrm{Ri}$ & 5 & $3648 \times 2736$ & 700 \\
\hline$\sum$ & 10 & 37 & & 5700 \\
\hline \hline
\end{tabular}




\begin{tabular}{|c|c|c|c|c|c|c|c|c|c|c|c|c|}
\hline & & \multicolumn{10}{|c|}{ Inspected images } & \multirow[b]{2}{*}{ Avg. } \\
\hline & & $\mathrm{Cn}$ & $\mathrm{Cs}$ & $\mathrm{F}$ & N1 & N2 & $\mathrm{O}$ & $\mathrm{Pa}$ & $\mathrm{Pe}$ & $\operatorname{Pr}$ & $\mathrm{Ri}$ & \\
\hline \multirow{11}{*}{$\mathcal{H}_{0}$} & $\mathrm{Cn}$ & 100 & $*$ & $*$ & $*$ & $*$ & $*$ & $*$ & $*$ & $*$ & $*$ & \\
\hline & Cs & * & 99.82 & $*$ & $*$ & $*$ & $*$ & $*$ & $*$ & $*$ & * & \\
\hline & $\mathrm{F}$ & $*$ & $*$ & 91.55 & $*$ & $*$ & $*$ & $*$ & $*$ & $*$ & $*$ & \\
\hline & N1 & $*$ & $*$ & $*$ & 100 & $*$ & $*$ & $*$ & $*$ & $*$ & $*$ & \\
\hline & $\mathrm{N} 2$ & $*$ & $*$ & $*$ & $*$ & 100 & $*$ & $*$ & $*$ & $*$ & $*$ & \\
\hline & $\mathrm{O}$ & $*$ & $*$ & $*$ & $*$ & $*$ & 97.25 & $*$ & $*$ & $*$ & $*$ & \\
\hline & $\mathrm{Pa}$ & $*$ & $*$ & $*$ & $*$ & $*$ & $*$ & 98.57 & $*$ & $*$ & $*$ & \\
\hline & $\mathrm{Pe}$ & $*$ & $*$ & $*$ & $*$ & $*$ & $*$ & $*$ & 92.21 & $*$ & * & \\
\hline & $\operatorname{Pr}$ & $*$ & $*$ & $*$ & $*$ & $*$ & $*$ & $*$ & $*$ & 100 & * & \\
\hline & \multirow[t]{2}{*}{$\mathrm{Ri}$} & $*$ & $*$ & $*$ & $*$ & $*$ & $*$ & $*$ & $*$ & $*$ & 98.33 & \\
\hline & & & & & & & & & & & & 97.77 \\
\hline
\end{tabular}

Table 2: Performance of proposed detector (the symbol* represents values smaller than $2 \%$ )

$\mathcal{H}_{0}$ against the others, thus the sum of a class may not yield $100 \%$. Therefore, it could lead to a scenario that an image is detected taken by at least two camera models. To deal with this scenario, the GLRT $\widehat{\delta}_{1}^{\star}$ could be performed on the camera models of conflict to better classify the inspected images.

The comparison with prior-art detectors includes the Support Vector Machine (SVM)-based detector that has already been performed on the Dresden database [32]. This detector is based on the statistical difference in natural images that are captured by different camera models. To capture this statistical difference, the SVM-based detector uses 46 different features. The feature set includes three main groups: color features describing the color reproduction of a camera model, wavelet statistics quantifying sensor noise and image quality metrics measuring sharpness and noise. The reader is referred to [36] for more details of this feature set. Moreover, the SVM might be the most popular choice among many existing powerful machine learning algorithms for supervised classification. The SVM-based detector has used $60 \%$ of the images of one device per model for training and all images of the remaining devices for testing. The detection performance of the SVM-based detector is shown in Table 3. The proposed detector $\widehat{\delta}_{2}^{\star}$ is almost equivalent to the SVM-based detector.

Furthermore, the PRNU-based detector [14] is also performed for reference. The PRNU is considered as the reliable fingerprint for image origin identification. Note that this detector is only performed on one device per model. Its detection performance is shown in Table 4. It is worth noting that the SVM-based and PRNU-based detectors cannot guarantee a prescribed false alarm rate. The proposed detector is not only able to guarantee a prescribed false alarm rate but also ensure a high detection performance. This emphasizes the strength of the proposed approach that is based on a relevant parametric image model to design a statistical test within hypothesis testing framework.

Besides, we also conduct the detector based on DCT coefficients statistics that is provided in our previous work [22] for comparison. The results of this statistical test are presented in the Table 5. The second GLRT is performed so that these two detectors are in the same scenario. In general, the DCT-based detector is less powerful and provides an 


\begin{tabular}{|c|c|c|c|c|c|c|c|c|c|c|c|c|}
\hline & & \multicolumn{10}{|c|}{ Inspected images } & \multirow[b]{2}{*}{ Avg. } \\
\hline & & $\mathrm{Cn}$ & Cs & F & N1 & $\mathrm{N} 2$ & $\mathrm{O}$ & $\mathrm{Pa}$ & $\mathrm{Pe}$ & $\operatorname{Pr}$ & $\mathrm{Ri}$ & \\
\hline \multirow{11}{*}{$\mathcal{H}_{0}$} & $\mathrm{Cn}$ & 98.26 & $*$ & $*$ & 2.61 & $*$ & $*$ & $*$ & $*$ & $*$ & $*$ & \\
\hline & $\mathrm{Cs}$ & $*$ & 99.71 & $*$ & 2.77 & $*$ & $*$ & $*$ & $*$ & $*$ & $*$ & \\
\hline & $\mathrm{F}$ & $*$ & $*$ & 99.94 & $*$ & $*$ & $*$ & $*$ & $*$ & $*$ & $*$ & \\
\hline & N1 & $*$ & $*$ & $*$ & 93.73 & $*$ & $*$ & $*$ & $*$ & * & $*$ & \\
\hline & $\mathrm{N} 2$ & $*$ & $*$ & $*$ & $*$ & 84.57 & $*$ & $*$ & $*$ & $*$ & $*$ & \\
\hline & $\mathrm{O}$ & $*$ & $*$ & $*$ & $*$ & $*$ & 98.95 & $*$ & * & * & $*$ & \\
\hline & $\mathrm{Pa}$ & $*$ & $*$ & $*$ & $*$ & $*$ & $*$ & 97.31 & $*$ & $*$ & $*$ & \\
\hline & $\mathrm{Pe}$ & $*$ & $*$ & $*$ & $*$ & $*$ & $*$ & $*$ & 90.86 & $*$ & $*$ & \\
\hline & $\operatorname{Pr}$ & $*$ & $*$ & $*$ & $*$ & $*$ & $*$ & $*$ & $*$ & 98.42 & $*$ & \\
\hline & \multirow[t]{2}{*}{$\mathrm{Ri}$} & $*$ & $*$ & $*$ & $*$ & $*$ & $*$ & $*$ & $*$ & $*$ & 100 & \\
\hline & & & & & & & & & & & & 96.17 \\
\hline
\end{tabular}

Table 3: Performance of SVM-based detector [32]

important misclassification, see groups $(\mathrm{Cn}, \mathrm{N} 1, \mathrm{Pe})$. However, one can note that this DCT based GLRT provides for two models (Olympus 1050SW and Pentax A40, respectively denoted 'O' and 'Pe') a slightly higher power than the spatial domain based GLRT $\widehat{\delta}_{2}^{\star}$ proposed in this paper. This can be explained by different quantization tables used for those two specific models compared to other models used in this present numericaml experiments. (RC:Thanh Hai, peux tu confirmer ou apporter une autre explication ?)

\section{Conclusion}

This paper designs statistical tests for camera model identification from JPEG images within hypothesis testing framework. The approach is based on the generalized noise model that is more accurate to characterize a natural image acquired by a digital camera. The parameters $(\tilde{a}, \tilde{b}, \gamma)$ characterizing the generalized noise model are exploited as camera fingerprint for camera model identification. Based on the proposed image model, this paper studies the most powerful LRT and designs two GLRTs that can be straightforwardly applied in practice. The strength of the proposed approach is that statistical performance of the tests can be analytically established as well as they can warrant a prescribed false alarm rate while ensuring a high detection performance. Future researches can exploit this approach for image forgery detection, for instance detecting a forged region that is taken by a different camera model.

\section{Appendix A. Proof of Proposition 1}

Under hypothesis $\mathcal{H}_{j}$, it follows from $z_{k, i} \sim \mathcal{N}\left(\mu_{k}, \sigma_{k, j}^{2}\right)$ that

$$
\frac{\left(z_{k, i}-\mu_{k}\right)^{2}}{\sigma_{k, j}^{2}} \sim \chi_{1}^{2},
$$




\begin{tabular}{|c|c|c|c|c|c|c|c|c|c|c|c|c|}
\hline & & \multicolumn{10}{|c|}{ Inspected images } & \multirow[b]{2}{*}{ Avg. } \\
\hline & & $\mathrm{Cn}$ & Cs & F & N1 & N2 & $\mathrm{O}$ & $\mathrm{Pa}$ & $\mathrm{Pe}$ & $\operatorname{Pr}$ & $\mathrm{Ri}$ & \\
\hline \multirow{11}{*}{$\mathcal{H}_{0}$} & $\mathrm{Cn}$ & 100 & $*$ & $*$ & $*$ & $*$ & $*$ & $*$ & $*$ & $*$ & $*$ & \\
\hline & Cs & $*$ & 99.44 & $*$ & $*$ & $*$ & $*$ & $*$ & $*$ & $*$ & $*$ & \\
\hline & $\mathrm{F}$ & $*$ & $*$ & 73.81 & $*$ & $*$ & $*$ & $*$ & $*$ & $*$ & $*$ & \\
\hline & N1 & $*$ & $*$ & $*$ & 98.92 & $*$ & $*$ & $*$ & $*$ & $*$ & $*$ & \\
\hline & $\mathrm{N} 2$ & $*$ & $*$ & $*$ & $*$ & 100 & $*$ & $*$ & $*$ & $*$ & $*$ & \\
\hline & $\mathrm{O}$ & $*$ & $*$ & $*$ & $*$ & $*$ & 87.25 & $*$ & $*$ & $*$ & $*$ & \\
\hline & $\mathrm{Pa}$ & $*$ & $*$ & $*$ & $*$ & $*$ & $*$ & 94.71 & $*$ & $*$ & $*$ & \\
\hline & $\mathrm{Pe}$ & $*$ & $*$ & $*$ & $*$ & $*$ & $*$ & $*$ & 100 & $*$ & $*$ & \\
\hline & $\operatorname{Pr}$ & $*$ & $*$ & $*$ & $*$ & $*$ & $*$ & $*$ & $*$ & 96.65 & $*$ & \\
\hline & \multirow[t]{2}{*}{$\mathrm{Ri}$} & $*$ & $*$ & $*$ & $*$ & $*$ & $*$ & $*$ & $*$ & $*$ & 100 & \\
\hline & & & & & & & & & & & & 95.07 \\
\hline
\end{tabular}

Table 4: Performance of PRNU-based detector [14]

where $\chi_{1}^{2}$ denotes chi-square distribution with one degree of freedom. Consequently, we derive

$$
\begin{aligned}
\mathbb{E}_{\mathcal{H}_{j}}\left[\left(z_{k, i}-\mu_{k}\right)^{2}\right] & =\sigma_{k, j}^{2} \\
\operatorname{Var}_{\mathcal{H}_{j}}\left[\left(z_{k, i}-\mu_{k}\right)^{2}\right] & =2 \sigma_{k, j}^{4} .
\end{aligned}
$$

By taking expectation and variance of the $\operatorname{LR} \Lambda\left(z_{k, i}\right)$, the proof of Proposition 1 follows immediately.

\section{Appendix B. Proof of Theorem 1}

From (11), the statistical distribution of the normalized $\operatorname{LR} \Lambda^{\star}(\mathbf{Z})$ can be given as

$$
\begin{cases}\Lambda^{\star}(\mathbf{Z}) \stackrel{D}{\longrightarrow} \mathcal{N}(0,1) & \text { under } \quad \mathcal{H}_{0}, \\ \Lambda^{\star}(\mathbf{Z}) \stackrel{D}{\longrightarrow} \mathcal{N}\left(\frac{m_{1}-m_{0}}{\sqrt{v_{0}}}, \frac{v_{1}}{v_{0}}\right) & \text { under } \quad \mathcal{H}_{1} .\end{cases}
$$

Consequently, based on the definition the decision threshold $\tau^{\star}$, it follows that

$$
1-\alpha_{0}=\mathbb{P}_{\mathcal{H}_{0}}\left[\Lambda^{\star}(\mathbf{Z}) \leq \tau^{\star}\right]=\Phi\left(\tau^{\star}\right) .
$$

The decision threshold $\tau^{\star}$ can be obtained immediately

$$
\tau^{\star}=\Phi^{-1}\left(1-\alpha_{0}\right)
$$

Similarly, based on the definition of the power function in (6), the power function $\beta_{\delta^{\star}}$ can be given as

$$
\begin{aligned}
\beta_{\delta^{\star}} & =\mathbb{P}_{\mathcal{H}_{1}}\left[\Lambda^{\star}(\mathbf{Z}) \geq \tau^{\star}\right]=1-\mathbb{P}_{\mathcal{H}_{1}}\left[\Lambda^{\star}(\mathbf{Z}) \leq \tau^{\star}\right] \\
& =1-\Phi\left(\frac{\tau^{\star}-\frac{m_{1}-m_{0}}{\sqrt{v_{0}}}}{\sqrt{\frac{v_{1}}{v_{0}}}}\right)=1-\Phi\left(\frac{m_{0}-m_{1}+\tau^{\star} \sqrt{v_{0}}}{\sqrt{v_{1}}}\right) .
\end{aligned}
$$




\begin{tabular}{|c|c|c|c|c|c|c|c|c|c|c|c|c|}
\hline & & \multicolumn{10}{|c|}{ Inspected images } & \multirow[b]{2}{*}{ Avg. } \\
\hline & & $\mathrm{Cn}$ & Cs & F & N1 & $\mathrm{N} 2$ & $\mathrm{O}$ & $\mathrm{Pa}$ & $\mathrm{Pe}$ & $\operatorname{Pr}$ & $\mathrm{Ri}$ & \\
\hline \multirow{11}{*}{$\mathcal{H}_{0}$} & $\mathrm{Cn}$ & 100 & $*$ & $*$ & 22.32 & $*$ & $*$ & $*$ & 18.54 & $*$ & $*$ & \\
\hline & $\mathrm{Cs}$ & $*$ & 98.72 & $*$ & $*$ & $*$ & $*$ & $*$ & $*$ & $*$ & $*$ & \\
\hline & $\mathrm{F}$ & $*$ & $*$ & 92.21 & $*$ & $*$ & $*$ & $*$ & $*$ & $*$ & $*$ & \\
\hline & N1 & $*$ & $*$ & $*$ & 85.64 & $*$ & $*$ & $*$ & 6.75 & $*$ & $*$ & \\
\hline & N2 & $*$ & $*$ & $*$ & $*$ & 100 & $*$ & $*$ & $*$ & $*$ & $*$ & \\
\hline & $\mathrm{O}$ & $*$ & $*$ & $*$ & $*$ & $*$ & 96.11 & $*$ & $*$ & $*$ & $*$ & \\
\hline & $\mathrm{Pa}$ & $*$ & $*$ & $*$ & $*$ & $*$ & $*$ & 99.52 & $*$ & $*$ & $*$ & \\
\hline & $\mathrm{Pe}$ & $*$ & $*$ & $*$ & 8.93 & $*$ & $*$ & $*$ & 82.78 & $*$ & $*$ & \\
\hline & $\operatorname{Pr}$ & $*$ & $*$ & $*$ & $*$ & $*$ & $*$ & $*$ & $*$ & 95.44 & $*$ & \\
\hline & \multirow[t]{2}{*}{$\mathrm{Ri}$} & $*$ & $*$ & $*$ & $*$ & $*$ & $*$ & $*$ & $*$ & $*$ & 98.12 & \\
\hline & & & & & & & & & & & & 94.85 \\
\hline
\end{tabular}

Table 5: Performance of DCT-based detector [22]

\section{Appendix C. Proof of Proposition 2}

Since the variance of $\hat{\mu}_{k}$ is negligible, it is proposed to treat $\hat{\mu}_{k}$ as a constant $\mu_{k}$, i.e.

$$
\begin{aligned}
& \widehat{\Lambda}_{2}\left(z_{k, i}\right)=\frac{1}{2} \log \frac{f\left(\mu_{k} ; \tilde{a}_{0}, \tilde{b}_{0}, \gamma_{0}\right)}{f\left(\mu_{k} ; \hat{\tilde{a}}_{1}, \hat{\tilde{b}}_{1}, \gamma_{1}\right)} \\
& +\frac{f\left(\mu_{k} ; \hat{\tilde{a}}_{1}, \hat{\tilde{b}}_{1}, \gamma_{1}\right)-f\left(\mu_{k} ; \tilde{a}_{0}, \tilde{b}_{0}, \gamma_{0}\right)}{2 f\left(\mu_{k} ; \hat{\tilde{a}}_{1}, \hat{\tilde{b}}_{1}, \gamma_{1}\right) f\left(\mu_{k} ; \tilde{a}_{0}, \tilde{b}_{0}, \gamma_{0}\right)}\left(z_{k, i}-\mu_{k}\right)^{2} .
\end{aligned}
$$

Since the ML estimates $\left(\hat{\tilde{a}}_{1}, \hat{\tilde{b}}_{1}\right)$ are consistent, it follows from the Slutsky's theorem [19, theorem 11.2.11] that the mathematical expectation of the GLR $\widehat{\Lambda}_{2}\left(z_{k, i}\right)$ does not chagne.

Meanwhile, the variance of $f\left(\mu_{k} ; \hat{\tilde{a}}_{1}, \hat{\tilde{b}}_{1}, \gamma_{1}\right)$ under each hypothesis $\mathcal{H}_{j}$ is given by

$$
\begin{aligned}
\operatorname{Var}_{\mathcal{H}_{j}}\left[f\left(\mu_{k} ; \hat{\tilde{a}}_{1}, \hat{\tilde{b}}_{1}, \gamma_{1}\right)\right]= & \operatorname{Var}_{\mathcal{H}_{j}}\left[\frac{1}{\gamma_{1}^{2}} \hat{\tilde{a}}_{1} \mu_{k}^{2-\gamma_{1}}+\frac{1}{\gamma_{1}^{2}} \hat{\tilde{b}}_{1} \mu_{k}^{2-2 \gamma_{1}}\right] \\
= & \frac{\mu_{k}^{4-2 \gamma_{1}}}{\gamma_{1}^{4}} \operatorname{Var}_{\mathcal{H}_{j}}\left[\hat{a}_{1}\right]+\frac{\mu_{k}^{4-2 \gamma_{1}}}{\gamma_{1}^{4}} \operatorname{Var}_{\mathcal{H}_{j}}\left[\hat{b}_{1}\right] \\
& +\frac{2 \mu_{k}^{4-3 \gamma_{1}}}{\gamma_{1}^{4}} \operatorname{Cov}_{\mathcal{H}_{j}}\left[\hat{\tilde{a}}_{1} ; \hat{\hat{b}}_{1}\right] \\
= & \frac{\mu_{k}^{4-2 \gamma_{1}}}{\gamma_{1}^{4}} \sigma_{\tilde{a}_{1}}^{2}+\frac{\mu_{k}^{4-4 \gamma_{1}}}{\gamma_{1}^{4}} \sigma_{\tilde{b}_{1}}^{2} \\
& +2 \frac{\mu_{k}^{4-3 \gamma_{1}}}{\gamma_{1}^{4}} \sigma_{\tilde{a}_{1} \tilde{b}_{1} .}
\end{aligned}
$$


Based on the Delta method [19, theorem 11.2.14], we derive

$$
\begin{aligned}
\operatorname{Var}_{\mathcal{H}_{j}}\left[\log \left(\frac{f\left(\mu_{k} ; \tilde{a}_{0}, \tilde{b}_{0}, \gamma_{0}\right)}{f\left(\mu_{k} ; \hat{\tilde{a}}_{1}, \hat{\tilde{b}}_{1}, \gamma_{1}\right)}\right)\right] & =\operatorname{Var}_{\mathcal{H}_{j}}\left[\log \left(f\left(\mu_{k} ; \hat{\tilde{a}}_{1}, \hat{\tilde{b}}_{1}, \gamma_{1}\right)\right)\right] \\
& \approx \frac{\operatorname{Var}_{\mathcal{H}_{j}}\left[f\left(\mu_{k} ; \hat{\tilde{a}}_{1}, \hat{\tilde{b}}_{1}, \gamma_{1}\right)\right]}{f^{2}\left(\mu_{k} ; \tilde{a}_{1}, \tilde{b}_{1}, \gamma_{1}\right)}
\end{aligned}
$$

and

$$
\begin{aligned}
& \operatorname{Var}_{\mathcal{H}_{j}}\left[\frac{f\left(\mu_{k} ; \hat{\tilde{a}}_{1}, \hat{\tilde{b}}_{1}, \gamma_{1}\right)-f\left(\mu_{k} ; \tilde{a}_{0}, \tilde{b}_{0}, \gamma_{0}\right)}{f\left(\mu_{k} ; \hat{\tilde{a}}_{1}, \hat{\tilde{b}}_{1}, \gamma_{1}\right) f\left(\mu_{k} ; \tilde{a}_{0}, \tilde{b}_{0}, \gamma_{0}\right)}\right] \\
& =\operatorname{Var}_{\mathcal{H}_{j}}\left[\frac{1}{f\left(\mu_{k} ; \hat{\tilde{a}}_{1}, \hat{\tilde{b}}_{1}, \gamma_{1}\right)}\right] \approx \frac{\operatorname{Var}_{\mathcal{H}_{j}}\left[f\left(\mu_{k} ; \hat{\tilde{a}}_{1}, \hat{\tilde{b}}_{1}, \gamma_{1}\right)\right]}{f^{4}\left(\mu_{k} ; \tilde{a}_{1}, \tilde{b}_{1}, \gamma_{1}\right)}
\end{aligned}
$$

Consequently, based on the law of total variance, it follows that

$$
\begin{aligned}
\operatorname{Var}_{\mathcal{H}_{j}}\left[\widehat{\Lambda}_{2}\left(z_{k, i}\right)\right]= & \frac{1}{2}\left(\frac{1}{\sigma_{k, 0}^{2}}-\frac{1}{\sigma_{k, 1}^{2}}\right)^{2} \sigma_{k, j}^{4} \\
& +\frac{1}{4} \frac{\operatorname{Var}_{\mathcal{H}_{j}}\left[f\left(\hat{\mu}_{k} ; \hat{\tilde{a}}_{1}, \hat{\tilde{b}}_{1}, \gamma_{1}\right)\right]}{\sigma_{k, 1}^{4}} \\
& +\frac{3}{4} \frac{\operatorname{Var}_{\mathcal{H}_{j}}\left[f\left(\hat{\mu}_{k} ; \hat{\tilde{a}}_{1}, \hat{\tilde{b}}_{1}, \gamma_{1}\right)\right]}{\sigma_{k, 1}^{8}} \sigma_{k, j}^{4}
\end{aligned}
$$

The proof follows immediately.

\section{Appendix D. Proof of Theorem 2}

From (27), in virtue of the Slutsky's theorem, the statistical distribution of the normalized GLR $\widehat{\Lambda}_{2}^{\star}(\mathbf{Z})$ can be derived as

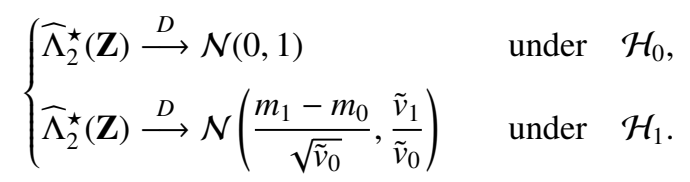

Based on the similar steps given in Appendix B, the proof follows immediately.

\section{References}

[1] M. C. Stamm, M. Wu, K. J. R. Liu, Information forensics: An overview of the first decade, IEEE Access 1 (2013) 167-200.

[2] G. L. Friedman, The trustworthy digital camera: restoring credibility to the photographic image, IEEE Trans. Consum. Electron. 39 (4) (1993) 905-910.

[3] C. I. Podilchuk, E. J. Delp, Digital watermarking: algorithms and applications, IEEE Signal Process. Mag. 18 (4) (2001) $33-46$.

[4] R. Ramanath, W. E. Snyder, Y. Yoo, M. S. Drew, Color image processing pipeline, IEEE Signal Process. Mag. 22 (1) (2005) $34-43$.

[5] J. Nakamura, Image Sensors and Signal Processing for Digital Still Cameras, CRC Press, 2005.

[6] K. S. Choi, E. Y. Lam, K. Wong, Source camera identification using footprints from lens aberration, in: Proc. SPIE Digit. Photography, vol. 6069 (2006), pp. 172-179. 
[7] A. Swaminathan, M. Wu, K. J. R. Liu, Nonintrusive component forensics of visual sensors using output images, IEEE Trans. Inf. Forensics Security 2 (1) (2007) 91-106.

[8] H. Cao, A. C. Kot, Accurate detection of demosaicing regularity for digital image forensics, IEEE Trans. Inf. Forensics Security 4 (4) (2009) 899-910.

[9] S. Gao, G. Xu, R. Hu, Camera model identification based on the characteristic of CFA and interpolation, in: Proc. Int. Workshop Digital Forensics Watermarking, vol. 7128 (2012), pp. 268-280.

[10] Y. Hu, C.-T. Li, X. Lin, B.-B. Liu, Camera model identification based on the characteristic of CFA and interpolation, in: Proc. Int. Conf. Intelligent Inf. Hiding Multimedia Signal Process., 2012, pp. 325-330.

[11] K. S. Choi, E. Y. Lam, K. K. Y. Wong, Source camera identification by JPEG compression statistics for image forensics, in: IEEE Region Conf. TENCON, 2006, pp. 1-4.

[12] J. Lukas, J. Fridrich, M. Goljan, Digital camera identification from sensor pattern noise, IEEE Trans. Inf. Forensics Security 1 (2) (2006) $205-214$.

[13] M. Chen, J. Fridrich, M. Goljan, J. Lukas, Determining image origin and integrity using sensor noise, IEEE Trans. Inf. Forensics Security 3 (1) (2008) 74-90.

[14] J. Fridrich, Digital image forensics, IEEE Signal Process. Mag. 26 (2) (2009) 26-37.

[15] C.-T. Li, Y. Li, Color-decoupled photo response non-uniformity for digital image forensics, IEEE Trans. Circuits Syst. Video Technol. 22 (2) (2012) 260-271.

[16] K. Kurosawa, K. Kuroki, N. Saitoh, CCD fingerprint method-identification of a video camera from videotaped images, in: Proc. IEEE Int. Conf. Image Process., vol. 3 (1999), pp. 537-540.

[17] T. Filler, J. Fridrich, M. Goljan, Using sensor pattern noise for camera model identification, in: Proc. IEEE Int. Conf. Image Process., 2008, pp. 1296-1299.

[18] C. Scott, Performance measures for Neyman-Pearson classification, IEEE Trans. Inf. Theory 53 (8) (2007) $2852-2863$.

[19] E. L. Lehmann, J. P. Romano, Testing Statistical Hypotheses, 3rd edition, Springer, New york, 2005.

[20] R. Cogranne, T. Denemark, J. Fridrich, Theoretical model of the FLD ensemble classifier based on hypothesis testing theory, in: Proc. IEEE. Int. workshop on Information Forensics and Security (WIFS), 2014, pp. 167-172.

[21] T. H. Thai, R. Cogranne, F. Retraint, Camera model identification based on the heteroscedastic noise model, IEEE Trans. Image Process. 23 (1) (2014) 250-263.

[22] T. H. Thai, F. Retraint, R. Cogranne, Camera model identification based on DCT coefficients statistics, Digit. Signal Process. 40 (2015) 88-100.

[23] T. H. Thai, R. Cogranne, F. Retraint, Camera model identification based on hypothesis testing theory, in: Proc. Eur. Signal Process. Conf., 2012, pp. 1747-1751.

[24] G. E. Healey, R. Kondepudy, Radiometric CCD camera calibration and noise estimation, IEEE Trans. Pattern Anal. Mach. Intell. 16 (3) (1994) 267-276.

[25] A. Foi, M. Trimeche, V. Katkovnik, K. Egiazarian, Practical Poissonian-Gaussian noise modeling and fitting for single-image raw-data, IEEE Trans. Image Process. 17 (10) (2008) 1737-1754.

[26] T. H. Thai, R. Cogranne, F. Retraint, Optimal detector for camera model identification based on an accurate model of dct coefficients, in: Multimedia Signal Processing (MMSP), Proc. of IEEE International Workshop on, 2014, pp. 1-6.

[27] T. H. Thai, R. Cogranne, F. Retraint, Statistical model of quantized DCT coefficients: Application in the steganalysis of Jsteg algorithm, IEEE Trans. Image Process. 23 (5) (2014) 1980-1993.

[28] T. H. Thai, R. Cogranne, F. Retraint, Steganalysis of jsteg algorithm based on a novel statistical model of quantized DCT coefficients, in: Image Processing (ICIP), Proc. of IEEE International Conference on, 2013, pp. 4427-4431.

[29] T. H. Thai, R. Cogranne, F. Retraint, Statistical model of natural images, in: Proc. IEEE. Int. Conf. Image Process., 2012, pp. 2525-2528.

[30] A. D. Ker, P. Bas, R. Böhme, R. Cogranne, S. Craver, T. Filler, J. Fridrich, T. Pevný, Moving steganography and steganalysis from the 
laboratory into the real world, in: Proc. of ACM workshop on Information hiding and multimedia security, IH\&MMSec, 2013, pp. 45-58.

[31] T. H. Thai, F. Retraint, R. Cogranne, Generalized signal-dependent noise model and parameter estimation for natural images, Signal Processing 114 (2015) 164-170.

[32] T. Gloe, R. Bohme, The Dresden image database for benchmarking digital image forensics, in: Proc. ACM Symposium Applied Comput., vol. 2 (2010), pp. 1585-1591

[33] W. Pennebaker, J. Mitchell, JPEG Still Image Compression Data, Springer, 1992.

[34] M. A. Robertson, R. L. Stevenson, DCT quantization noise in compressed images, IEEE Trans. Circuits Syst. Video Technol. 15 (1) (2005) 27-38.

[35] J. A. Nelder, R. Mead, A simplex method for function minimization, Comput. J. 7 (1965) 308-313.

[36] T. Gloe, K. Borowka, A. Winkler, Feature-based camera model identification works in practice, in: Proc. Int. Workshop Inf. Hiding, Vol. 5806, 2009, pp. 262-276. 